difficult. Current medications and medical history was stated in $75.8 \%$ and $76.9 \%$ of the letters respectively. The past psychiatric history and family history was only stated in $28.6 \%$ and $6.6 \%$ of the letters, despite being of obvious importance. Social circumstances were mentioned in $53.8 \%$ of the letters. The Mini-Mental State Examination results and blood tests were recorded in only $13.2 \%$ of referral letters. The letters showed that in $90 \%$ of patients no X-rays were done, with only $6.6 \%$ of patients having computed tomography brain scan and $3.3 \%$ of patients having magnetic resonance imaging completed.

This audit showed that many general practice referral letters are missing basic information that can compromise the initial assessment of the patients. The letters should contain enough information to ensure that patients are managed safely and effectively.

*Deirdre Hussey Honorary Lecturer in Psychiatry for Later Life, University College Hospital Galway, Co. Galway, Ireland, email: dhussey2@gmail.com,

Kevin Foy Senior Registrar in Psychiatry for Later Life, Karena Meehan Consultant Psychiatrist in Psychiatry for Later Life, University College Hospital Galway, Co. Galway, Ireland

doi: 10.1192/pb.33.4.154b

\section{Internet electroconvulsive therapy}

While undertaking my routine electroconvulsive therapy (ECT) clinic, I asked the anaesthetic nurse what she thought about the treatment. She said she would never have ECT because of all the 'things on the internet'. Out of curiosity, I did some basic searches on the internet about ECT. Worryingly, the idea that ECT is barbaric is all over the internet. There are harrowing accounts of ECT therapy so-called 'survivors' (http://endofshock. $\mathrm{com} /$ ). There are also some complete online video tutorials explaining why ECT should not be used (www.youtube.com/ watch?v=WBBtH14jEPI). One particularly concerning view is that put forward by actor Tom Cruise (www.youtube.com/ watch?v=TTr4F-5U29Q). These very public attacks by anti-psychiatry groups which centre on coercive practices and memory impairment cannot be ignored.

There have been many anti-psychiatric groups. As explained by Fink, 'In their early history they were led by scholars. For example Thomas Sasz, who felt that psychiatrists were used as a form of social control by government.. ${ }^{1}$ In modern times, many anti-psychiatry groups are led by patients. Anyone can find numerous antiECT websites and videos on the internet, and as clinicians we need to be aware that many of our patients could be visiting these sites or could have their own blogs.
We need to actively provide alternative information and resources to give patients a fairer view of ECT to help them make the best decision. The website www. patient.co.uk might be a good place for such information.

FINK, M. (1999) Electroshock: Restoring the Mind. Oxford University Press.

Neeraj Joshi CT1, Greater Manchester West Mental HealthTrust, email: neerajjoshi81@yahoo. co.uk

doi: 10.1192/pb.33.4.155

\section{Complementary and alternative treatments in psychiatric out-patients}

The use of complementary or alternative medicines or treatments in the general population is high - in the UK some $46 \%$ can be expected to use one or more in their lifetime (Bishop \& Lewith, 2008) Despite the popularity of alternative treatments, we were unable to find any data examining usage among psychiatric patients. To investigate this, we conducted a survey of out-patient attenders at our general psychiatry clinics; 87 consecutively attending patients were asked about their use of alternative therapies.

We found that $8(9 \%)$ patients were using complementary or alternative treatments: 3 aromatherapy oils, 1 oil of evening primrose, 1 chondroitin, 1 homeopathy, 1 Reiki therapy and 1 patient using a compound called Adutwumwaa Bitters. This preparation contains rauwolfia vomitoria, from which reserpine is obtained. Reserpine can cause depression through monamine depletion in synaptic vesicles.

We advocate that psychiatrists should routinely ask about the use of alternative treatments when assessing patients, as often patients do not volunteer this information to their doctors (Kamerow, 2007). It is possible that certain preparations or therapies may interact with medical treatments. This may contribute to the development, or exacerbation, of a psychiatric disorder.

BISHOP, F. L. \& LEWITH, G.T. (2008) Who uses CAM? A narrative review of demographic characteristics and health factors associated with CAM. Evidence-Based Complementary and Alternative Medicine, 13 March, doi:101093/ecam/nen023.

KAMEROW, D. (2007) Wham bam thank you CAM. $B M J, 335,647$

Richard Sankar ST2 trainee in psychiatry Moorgreen Hospital, Southampton, *Peter Childs Consultant Psychiatrist, Hawthorn Lodge, Moorgreen Hospital, Botley Road, West End Southampton SO30 3JB, email: peter.childs@ hantspt-sw.nhs.uk

doi: 10.1192/pb.33.4.155a

\section{Trust services for psychiatrists victimised by stalkers}

Between 2 and $15 \%$ of Western populations report being stalked, depending on the stalking definition used (Tjaden \& Thoennes, 1998; Walby \& Allen, 2004). Doctors and other healthcare professionals are at higher than average risk (Lion \& Herschler, 1998; Pathé et al, 2002 Purcell et al, 2005). The high prevalence, and distressing and dangerous nature of stalking are widely reported, but services for victims of stalking remain patchy. We examined what help mental health trusts provide for psychiatrist employees victimised in this way, and the potential demand for support from the Royal College of Psychiatrists.

In a study conceived and designed with the help of Drs Edward Petch and David Reiss, we sent questionnaires to the medical directors of all 115 statutory mental health service providers in the UK, and followed-up non-responders after 4 months: 65 (57\%) responded. All but one respondent said their organisation would help psychiatrist employees who were victims of work-related stalking and 38 said they would also help with stalking that was not work-related. For workrelated stalking, the most common types of help offered were: discussion with a manager ( $n=36$ organisations); liaison with the police $(n=31)$; discussion with the clinical team or educational supervisor $(n=30)$; and support from the occupational health service $(n=30)$. Less common were legal services $(n=17)$; staff counselling or similar psychological support ( $n=17)$; changing the patient's care team $(n=6)$; and psychological support sourced externally $(n=4)$. Other types of help were reported by 14 organisations and included: financial support for security measures, advice from a trust specialist such as a security advisor or a human resources advisor, or from a forensic psychiatrist. The wide variation in responses, with many respondents not mentioning psychological support and very few mentioning practical interventions, indicates that locally available resources are inconsistent and that a central source of expertise, such as one provided by the College, might be beneficial.

Less than half $(n=27)$ of the respondents thought a College service for stalking victims would be useful, 19 thought it would not be useful and 19 were unsure. Those who supported a College service thought it should provide: practical advice ( $n=25$ respondents); psychological support $(n=17)$ advice to the employer $(n=8)$; legal support $(n=1)$; and links with other Royal 